\section{Renaissance in laser spectroscopy}

Frontiers in Laser Spectroscopy. (Les Houches 1975 Session XXVII) Vol. 1. Pp. 472. Vol. 2. Pp. 473-907. Edited by Roger Balian, Serge Haroche and Sylvain Liberman. (North-Holland: Amsterdam, New York and Oxford, 1977.) Dfl. $280 ; \$ 114.50$.

THESE two volumes present the proceedings of the Les Houches Theoretical Physics Summer School held in July 1975 on the use of lasers in atomic and molecular spectroscopy. The stated aim of the school was to "give a general presentation of the theoretical background necessary to understand light-matter interaction problems, and to present a review of the various new methods in which lasers are now being used to improve our knowledge about atoms, molecules and even about nuclei." Underlying these new methods are the recent exploitation of tunability, of saturation and of coherent transients which have contributed so much of late to the renaissance in spectroscopy. The level of presentation of the proceedings tends to assume previous knowledge at about first-year graduate student level of quantum optics and quantum electronics, and the scope of the discussion is very wide with most of the actively-pursued topics in the field at least mentioned.

Cohen-Tannoudji presents a marginally telegraphic overview of the basic phenomena involved in the interaction of atoms with strong resonant fields, and his thoughtful lectures contain many insights into fundamental problems involving the non-linear saturation of fluorescent emission. This is followed in Volume 1 by lectures on laser theory (Sargent); transient and steady-state response of two- and threelevel systems, including a very useful discussion of the semiclassical theory of superradiance (Feld); the theory and observation by stark-switching of optical nutation, free-induction decay, photon echoes and two-photon transitions (Brewer); and single-atom and cooperative quantum beats (Stenholm). These lectures form a coherent and well-presented review of a physically well-defined area.

In Volume 2, this coherence is lost to a certain extent by an attempt to cover a very wide range of techniques and specific applications. The use of lasers in very high resolution spectroscopy of molecules is discussed by Lehmann, Oka, Kelley and Javan. Letokhov discusses the coupling be- tween electronic and nuclear degrees of freedom in laser-driven transitions and the so-far purely theoretical subject of "laser-nuclear spectroscopy."

Finally, one of the topics which ensures adequate funding, namely isotope separation, is admirably discussed by Letokhov. Also included in these volumes are short seminars by Series, Walther, Cagnac, Haroche, Liberman, Toschek, Mukamel and Monchalin, which are useful as illustrations of particular research problems, although a few should have been (and some have been) published in the primary literature.

Some of these lectures are rather experimental in tone; these though no less valuable, do not quite fit the label of "Ecole d'Été de Physique Theorique." Indeed, an interesting feature

\section{Explosion in myelin research}

Myelin. Edited by P. Morell. Pp. xxii+ 531. (Plenum: New York and London, 1977). \$47.40.

DURING the past decade there has been an explosive increase in research on myelin. Now with the publication of a major book on the subject, it is possible to see why. Myelin is one of the most abundant of biological membranes constituting about $50 \%$ of the dry weight of the white matter, from which relatively pure fractions can be easily isolated by centrifugation. Such procedures are described in the book together with tabulated data of lipid and protein analysis of myelin fractions of central and peripheral nerve. Extensive biophysical data (for example, X-ray and neutron diffraction) has been correlated with the chemistry and the ingenious use of probe analysis. As a result, we probably know more about the molecular architecture of myelin than many other biological membranes.

The first chapter contains a great deal of impressive and well illustrated information on the morphology of the myelin-formative glial or Schwann cells. Although little is known about the mechanisms controlling the transition stage between glial plasma membrane and the fully mature compact myelin lamellae, the glial cell must, during the period of myelination, have a remarkable synthetic activity, for it seems to be capable of making three times its weight of myelin per day. The myelinating brain therefore provides a unique system for studying membrane biosynthesis. Some of the relevant precursor-product labelling of this field is the ability of the best practitioners (some of them contributing to these volumes) to cross the experimental-theoretical divide to the great benefit of both sides. Since there were only 42 "pupils" attending the school, one would hope that the publication of the proceedings would give a wider circulation to these valuable lectures. The price of these volumes seems designed to ensure that this will not be so. I doubt the value of such lavish publication of conference and Summer school proceedings in hardback textbook format, with its concomitant delay and inflated cost.

Peter Knight

Peter Knight is Jubilee Research Fellow in Physics at Royal Holloway College, University of London, UK.

studies and the so far equivocal attempts at isolation of intermediary 'myclin-like' fractions from developing brain are critically discussed by different contributors.

Study of the biology of myelin is, however, of more than academic interest for in several neurological diseases there is damage to the white matter with accompanying loss of myelin. In the otherwise excellent chapters on neuropathology and neurology of myelin diseases more space could have usefully been devoted to multiple sclerosis, since this is one of the commonest neurological diseases.

Other chapters cover chemical pathology, experimental pathology and animal models of genetic myelin disorder. One of the most important features of myelin pathology relates to the pronounced immunological response to the protein antigens and lipid haptens (for example, cerebroside). This subject is especially relevant following the recent discovery of a relaxing and remitting form of experimental allergic encephalitis.

It will be evident that the whole intriguing subject of the biology of myelin is well covered in this book and it can be recommended as a readable and authoritative account of the subject.

Although the book is on a specialist subject it can be recommended to the general reader who might thereby be encouraged to utilise some of the basic concepts and discoveries on myelin in other fields of biology

A. N. Davison

A. N. Davison is Professor of Neurochemistry at the Institute of Neurology and Head of the Department of Neurochemistry and Chemical Pathology. National Hospitals for Nervous Diseases, London, UK. 